\title{
An atypical presentation of sinus mucopyocele in a pediatric cystic fibrosis patient
}

\author{
This article was published in the following Dove Press journal: \\ Clinical Ophthalmology \\ 6 May 2015 \\ Number of times this article has been viewed
}

\section{Elan Horesh \\ Andrew A Colin \\ Roy Casiano \\ Sara T Wester}

Bascom Palmer Eye Institute, University of Miami Miller School of Medicine, Miami, FL, USA
Correspondence: Sara Wester Bascom Palmer Eye Institute, University of Miami Miller school of Medicine, 900 NW 17th Street, Miami, FL 33136, USA

Tel +l 3053266086

Email swester2@med.miami.edu

\begin{abstract}
This case report details an association of chronic allergic conjunctivitis and respiratory tract colonization in a cystic fibrosis (CF) patient due to an ethmoidal mucocele infected with Escherichia coli. A 3-year-old CF patient presented for evaluation with complaints of chronic periocular erythema, conjunctival injection, and irritation for 2 years. He was treated for presumed allergic conjunctivitis with no improvement and continued to have overall worsening of symptoms on the right greater than the left eye in a waxing and waning pattern. On presentation to the Bascom Palmer Eye Institute, he was noted to have telecanthus and prominent erythema in the region of the medial canthus. Orbital imaging disclosed a mucocele in the right ethmoid sinus. The patient underwent functional endoscopic sinus surgery, with successful marsupialization of the ethmoidal mucocele, which was found on culture to be infected with $E$. coli. Postoperatively with continuous pulmonary care, the patient remains free of allergic conjunctivitis and $E$. coli colonization of the upper airway. This case highlights the importance of analyzing the adjacent sinus in patients with chronic, relapsing allergic conjunctivitis refractory to medical management, particularly in patients with underlying systemic diseases such as CF.
\end{abstract}

Keywords: allergic conjuncitivitis, Escherichia Coli, cystic fibrosis, mucocele

\section{Introduction}

Allergic conjunctivitis is the most common type I IgE-mediated hypersensitivity reaction to environmental allergens involving the ocular surface, affecting approximately $25 \%$ of the general population and $30 \%$ of allergy-predisposed children. ${ }^{1}$ Symptoms of allergic conjunctivitis include ocular pruritus, tearing, conjunctival edema, hyperemia, watery discharge, burning, and photophobia. ${ }^{2}$ Treatment of allergic conjunctivitis involves topical and sometimes oral therapy as well as allergen avoidance. The current literature is limited in terms of the association between sinus disease and chronic allergic conjunctivitis. ${ }^{3,4}$ While in other ophthalmic symptomatology (proptosis, epiphora, deep orbital pain, double vision), ${ }^{5-8}$ physicians often look to the adjacent sinus for the etiology, such an analysis is often overlooked in the patient with severe, unremitting allergic conjunctivitis refractory to traditional medical management. In particular, paranasal sinus mucocele has not been well documented as a possible cause of conjunctivitis, particularly in children. To our knowledge, there is only one case reported of a mucocele leading to chronic blepharoconjunctivitis and this was in an adult patient (the adult population is more likely to have mucoceles than the pediatric population). ${ }^{8}$ More common ophthalmic symptoms of these benign, epithelial-lined, mucus filled sacs include proptosis, visual disturbances, motility defects, and pain due to mass effect. ${ }^{3-8}$ Mucoceles have been documented in association with systemic diseases, such as cystic fibrosis (CF), with a prevalence of $16 \%$ in cases of $\mathrm{CF}$ with symptoms of chronic upper airway disease. In addition, patients 
with CF generally have a large number of bacteria in their sputum and nasal secretions ${ }^{9}$ and therefore have a higher risk of infection (mucopyocele). ${ }^{10}$ In cases of infection, drainage of the mucopyocele is indicated to ensure eradication of the organism that can have significant pulmonary consequences. Identification and diagnosis of the mucocele is critical in helping maintain the pulmonary health of such patients, which can be difficult in atypical cases without symptoms of nasal obstruction. We present herein a case of a 3-year-old $\mathrm{CF}$ patient misdiagnosed for 2 years with chronic allergic conjunctivitis whose symptoms were caused by an ethmoidal mucopyocele infected with Escherichia coli.

\section{Case report}

A 3-year-old boy under pediatric pulmonary care for $\mathrm{CF}$ presented to the Bascom Palmer Eye Institute, University of Miami, with a 2-year history of chronic bilateral conjunctival inflammation, mild epiphora, and irritation. Despite bilateral disease, the symptoms were significantly worse in the right eye than the left. The patient was evaluated in multiple subspecialty ophthalmology clinics both in the United States and internationally and in each, he was diagnosed with allergic conjunctivitis. His parents denied symptoms of nasal obstruction, other than snoring, which had been present prior to the onset of the eye symptoms. No cultures had been obtained from the conjunctiva. Treatment regimens, including emergency room treatment, involved the use of topical anti-allergy drops such as olopatadine (Patanol) which improved the swelling only temporarily. He was referred to us for further evaluation. Clinical examination disclosed erythema in the medial aspect of the orbit, lower lid greater than upper eyelid, a palpable prominence under the medial canthal tendon and in the region of the nasolacrimal sac, and telecanthus on the right (Figure 1).

Ocular examination was notable for significant papillary conjunctivitis of the right eye. Extraocular motility was full and the pupils were reactive OU without afferent pupillary defect. He had a normal tear lake and no mucopurulent reflex with compression of the nasolacrimal sac. The rest of his examination was within normal limits.

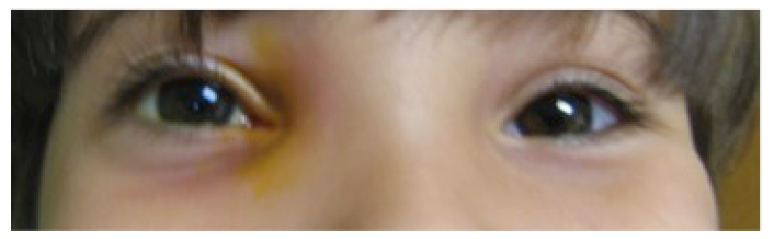

Figure I External photograph discloses a palpable prominence in the medial canthal area with surrounding erythema.
Past medical history was notable for CF; pancreatic insufficiency had been appropriately managed with pancreatic enzymes. Additionally, he was noted to have chronic airway (deep throat culture based) colonization with E. coli, an organism seen infrequently in young children with CF. Despite multiple attempts to eradicate it with nebulized tobramycin and oral antibiotics, the organism persisted. A decision was made against long-term antibiotics, as $E$. coli is not considered to be a Gram-negative organism that predicts an adverse pulmonary outcome in patients with CF. The patient was continuously followed by pulmonology to monitor his respiratory status.

Magnetic resonance imaging (MRI) of the brain was obtained to further evaluate the medial canthal prominence. In the orbits, an area of mass effect was seen in the canthal region of the right orbit. This area extended inferiorly to the level of the inferior turbinate and involved a large area of the ethmoid sinus on the right side. Mass effect also caused bowing of both the lamina papyracea and nasal septum. The mass had high signal intensity on T1 and low signal on T2 weighted images, and there was ring-like enhancement (Figure $2 \mathrm{~A}-\mathrm{C}$ ), most consistent with an ethmoidal mucocele.

The patient underwent functional endoscopic sinus surgery at the University of Miami with marsupialization of the right ethmoidal mucocele (Figure $3 \mathrm{~A}$ and B), which was later confirmed by culture to be infected with E. coli. After post-operative follow-up and continuous pulmonary care, the patient showed no symptoms of allergic conjunctivitis, the telecanthus and medial canthal mass resolved, and the upper airway has remained free of E. coli.

\section{Discussion}

The association between chronic conjunctivitis and mucocele in CF patients has not been well established in the literature but is an important consideration for the ophthalmologist, otolaryngologist, and pulmonologist managing these patients. Disease of the nose and paranasal sinuses occurs in almost all CF patients; most patients develop chronic sinusitis, and often bacterial infection thereof early in the course of their disease. While there is little recognition in the CF community of the presence of mucoceles, paranasal mucocele have in fact been documented to have a prevalence as high as $16 \%$ in patients with CF with symptoms of chronic upper airway disease who underwent otolaryngology evaluation. ${ }^{10}$ Interestingly, ocular or orbital findings are relatively rare in this population. Only one patient in this series presented with hyperemic conjunctiva, and this patient also had nasal symptoms including persistent purulent rhinorrhea and nasal obstruction. ${ }^{10}$ Other 

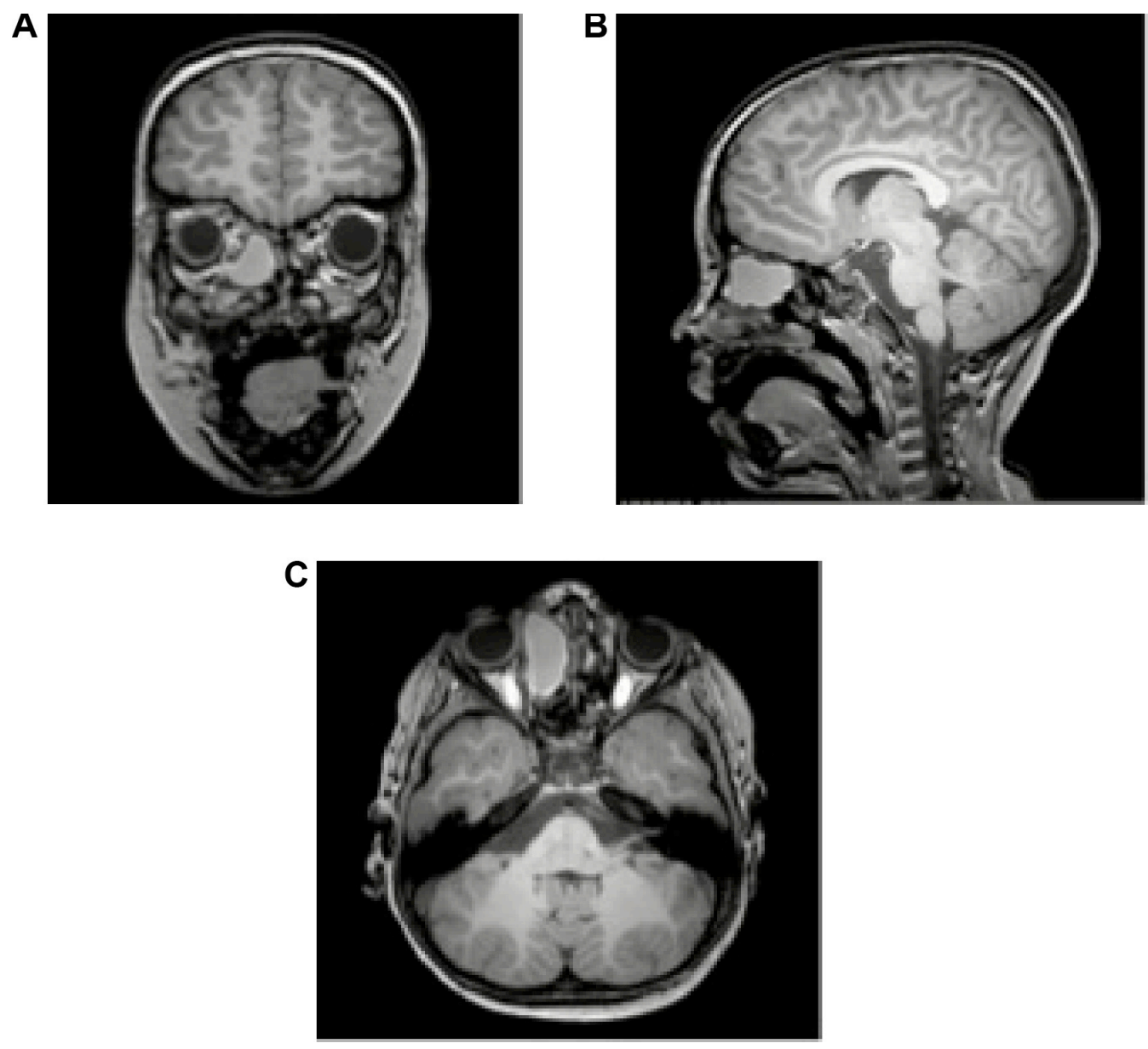

Figure 2 Magnetic resonance imaging (MRI) of patient demonstrating large ethmoidal mucocele on the right. Notes: (A) Coronal TI MRI, (B) sagittal TI MRI, (C) axial TI MRI.

case reports document mucoceles in the pediatric $\mathrm{CF}$ patients with the more typical findings of nasal obstruction..$^{11}$ Thus, in the common CF practice, conjunctival disease is not viewed as part of the presentation of a mucocele, and its presence would not alert the $\mathrm{CF}$ expert to a potential underlying contiguous infectious focus. More specifically, sinus disease is often not considered in a patient who presents with symptoms of periorbital or ocular allergic disease.

Our patient developed chronic, refractory, and asymmetric allergic conjunctivitis as well as recurrent E. coli
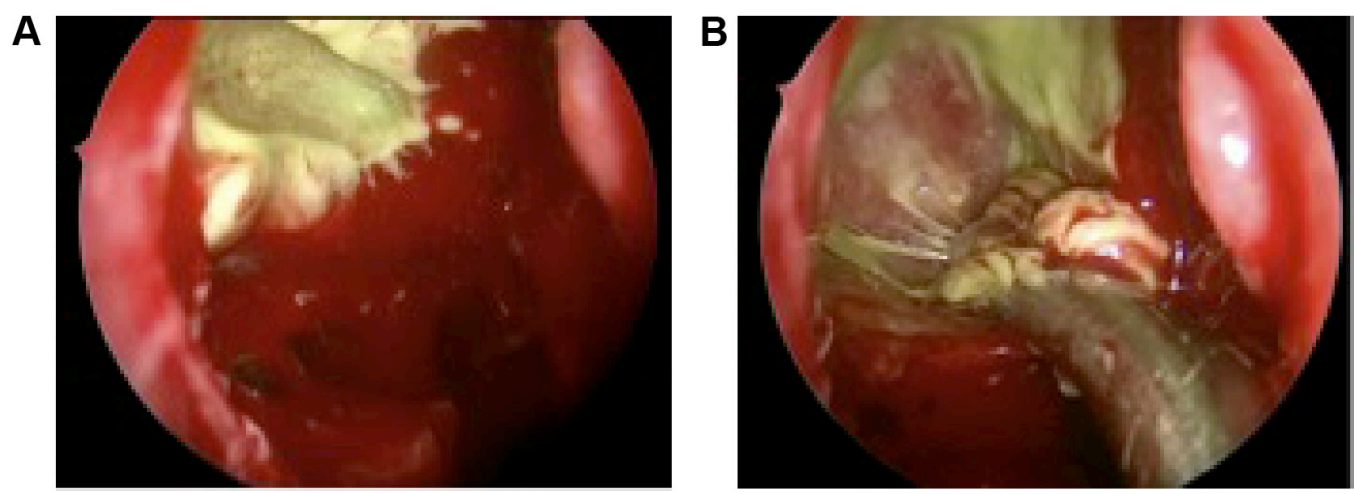

Figure 3 Images during FESS.

Notes: (A) View of sinus mucocele during FESS, (B) marsupialization of mucocele during FESS.

Abbreviation: FESS, functional endoscopic sinus surgery. 
colonization of the respiratory tract, both of which were manifestations of an ethmoidal mucopyocele. Both the recurrent airway $E$. coli colonization and symptoms of allergic conjunctivitis resolved post-functional endoscopic sinus surgery and he has remained stable. We hypothesize that the chronic low grade exposure to $E$. coli created an atypical allergic conjunctivitis. This hypothesis is supported by previous documentation of allergic conjunctivitis secondary to stimulus of the nasal mucosa, thought to be potentially related to various neuropeptides, cytokines, chemokines, chemotactic, and other factors. ${ }^{12}$ It is also possible that he in fact had a low-grade bacterial conjunctivitis given the significantly worse symptoms and signs on the eye ipsilateral to the mucocele, but this cannot be confirmed as cultures of the ocular surface were not performed. $E$. coli is a rare cause of ophthalmic infection, most commonly in the neonate causing infectious conjunctivitis or in immunosuppressed adults and is rarely found in the normal conjunctival flora. ${ }^{13}$ The important clinical factor is that his symptoms and exam, which were most consistent with allergic conjunctivitis, resolved completely after eradication of the mucocele.

It is always important to consider the adjacent sinuses when managing a patient with chronic periocular symptomatology, particularly allergic disease, even in pediatric cases. Of further important note is that in the specific condition of $\mathrm{CF}$, mucoceles are more common and are more likely to be infected (ie, mucopyoceles), while in otherwise normal patients they are almost uniformly sterile. This is an important distinction for the patient with $\mathrm{CF}$, since a chronic source of bacterial infection in the upper airway can become the source of infection spread to the lower airway, the latter infections being the major determinant of long-term outcome in such patients. Our case report highlights that chronic conjunctivitis in CF patients that is refractory to standard management should lead to a thorough evaluation including bacterial cultures and in some cases neuroimaging to determine and properly treat a putative underlying cause.

Clinical Ophthalmology

\section{Publish your work in this journal}

Clinical Ophthalmology is an international, peer-reviewed journal covering all subspecialties within ophthalmology. Key topics include: Optometry; Visual science; Pharmacology and drug therapy in eye diseases; Basic Sciences; Primary and Secondary eye care; Patient Safety and Quality of Care Improvements. This journal is indexed on Submit your manuscript here: http://www.dovepress.com/clinical-ophthalmology-journal

\section{Acknowledgments}

This study was supported by NIH Center Core Grant P30EY014801, Research to Prevent Blindness Unrestricted Grant, Department of Defense (DOD-grant number W81XWH-09-1-0675 and grant number W81XWH-13-10048 ONOVA).

\section{Disclosure}

The authors report no conflicts of interest in this work.

\section{References}

1. Boguniewicz, Mark, and Donald Y.M. Leung. Ocular Allergies. Ed. Robert M. Kliegman, et al. Nelson Textbook of Pediatrics. Philadelphia, PA: Elsevier/Saunders, 2011. 809-811.

2. Uptodate.com [homepage on the internet]. Dana R. Allergic Conjunctivitis. Allergic conjunctivitis: Clinical manifestations and diagnosis. UpToDate. Available from: http://www.uptodate.com/contents/allergicconjunctivitis?source=search_result. Accessed March 28, 2015.

3. Ch'ng SW, Pillai MB, Morton C. Frontal sinus mucoceles presenting in the upper eyelid: an easily missed diagnosis. BMJ Case Rep. 2012:2012. pii: bcr0320125974.

4. Peng KL, Tsai CC, Kau HC, Kao SC, Hsu WM. Giant mucocele masquerading as chronic unilateral conjunctivitis. Eye (Lond). 2003;17(3):454-456.

5. Abrahamson IA Jr, Baluyot ST, Tew JM Jr, Scioville G. Frontal sinus mucocele. Ann Ophthalmol. 1979;11(2):173-178.

6. Nugent GR, Sprinkle P, Bloor BM. Sphenoid sinus mucoceles. J Neurosurg. 1970;32(4):443-451.

7. Malhotra R, Wormald PJ, Selva D. Bilateral dynamic proptosis due to frontoethmoidal sinus mucocele. Ophthal Plast Reconstr Surg. 2003; 19(2):156-157.

8. Gray RF, Barton RP. Pneumosinus dilatans as a cause of variable unilateral proptosis. J Laryngol Otol. 1978;92(7):623-625.

9. Hodson ME Hodson, Geddes DM. Cystic Fibrosis, Second edition. London: CRC Press; 2000.

10. Di Cicco M, Costantini D, Padoan R, Colombo C. Paranasal mucoceles in children with cystic fibrosis. Int J Pediatr Otorhinolaryngol. 2005;69(10):1407-1413.

11. Aubry K, Orsel S, Menetrey C, Bessède JP, Sauvage JP. Bilateral paranasal sinus mucopyoceles in a child with cystic fibrosis. Rev Laryngol Otol Rhinol (Bord). 2009;130(4-5):293-294.

12. Hom M, Bielory L. The anatomical and functional relationship between allergic conjunctivitis and allergic rhinitis. Allergy Rhinol (Providence). 2013;4(3):e110-e119.

13. Chen CJ, Starr CE. Epidemiology of gram-negative conjunctivitis in neonatal intensive care unit patients. Am J Ophthalmol. 2008;145(6): 966-970.

\section{Dovepress}

PubMed Central and CAS, and is the official journal of The Society of Clinical Ophthalmology (SCO). The manuscript management system is completely online and includes a very quick and fair peer-review system, which is all easy to use. Visit http://www.dovepress.com/ testimonials.php to read real quotes from published authors. 